\title{
OPTIMAL PARAMETERS FOR A DAMPED SINE-GORDON EQUATION
}

\author{
Junhong Ha And Semion Gutman
}

\begin{abstract}
In this paper a parameter identification problem for a damped sine-Gordon equation is studied from the theoretical and numerical perspectives. A spectral method is developed for the solution of the state and the adjoint equations. The Powell's minimization method is used for the numerical parameter identification. The necessary conditions for the optimization problem are shown to yield the bang-bang control law. Numerical results are discussed and the applicability of the necessary conditions is examined.
\end{abstract}

\section{Introduction}

In this paper, we study the identification problem for parameters $\alpha, \beta$ and $\delta$ appearing in the one-dimensional damped sine-Gordon equation

$$
\begin{aligned}
& y_{t t}+\alpha y_{t}-\beta y_{x x}+\delta \sin y=f(t, x), \quad(t, x) \in Q=(0, T) \times(0, L), \\
& y(t, 0)=y(t, L)=0, \quad t \in(0, T), \\
& y(0, x)=y_{0}(x) \text { and } y_{t}(0, x)=y_{1}(x), \quad x \in(0, L) .
\end{aligned}
$$

Equation (1) describes the dynamics of a Josephson junction driven by a current source taking into account the damping effect, see [1]. The sine-Gordon model has attracted an additional interest since it is known to exhibit chaotic behavior for certain values of the governing parameters $\alpha, \beta$ and $\delta$. For details we refer to $[8]$ and $[11]$.

Let $f \in L^{2}(Q), y_{0} \in H_{0}^{1}(0, L)$ and $y_{1} \in L^{2}(0, L)$. The identification problem for (1) consists in finding the parameters $\alpha, \beta$ and $\delta$ such that the solution of (1) exhibits the desired behavior. More precisely, let $P=\left\{q=(\alpha, \beta, \delta) \in R^{3} \mid \beta>\right.$ $0\}$ be equipped with the Euclidean norm. Let $P_{a d} \subset P$ be an admissible set of parameters. Define the cost functional $J(q)$ by

$$
J(q)=\int_{Q}\left[y(q ; t, x)-z_{d}(t, x)\right]^{2} d x d t, \quad q \in P_{a d},
$$

Received February 14, 2008.

2000 Mathematics Subject Classification. Primary 49J20, 49K20.

Key words and phrases. optimal control, necessary condition, bang-bang control law.

(C)2009 The Korean Mathematical Society 
where $z_{d}$ is a given function on $Q$. The data $z_{d}$ can be thought of as the targeted behavior of (1). The parameter identification problem for (1) with the objective function (2) is to find $q^{*}=\left(\alpha^{*}, \beta^{*}, \delta^{*}\right) \in P_{a d}$ satisfying

$$
J\left(q^{*}\right)=\inf _{q \in P_{a d}} J(q), P_{a d} \subset P .
$$

The solution map $q \rightarrow y(q)$ from $P$ into $C\left([0, T] ; L^{2}(0, L)\right)$ is continuous under proper initial conditions, see [5] or Section 3. Hence it is clear that the minimization problem (3) has at least one solution $q^{*}$, provided $P_{a d}$ is bounded and closed.

On the other hand, the minimizer $q^{*}$ can be characterized by the inequality

$$
D J\left(q^{*}\right)\left(q-q^{*}\right) \geq 0, \quad q \in P_{a d},
$$

where $D J\left(q^{*}\right)\left(q-q^{*}\right)$ denotes the Gâteaux derivative of $J(q)$ at $q=q^{*}$ in the direction of $q-q^{*}$. The existence of the Gâteaux derivative was proved by us in [5]. We refer to $[6,7]$ for pertubed-sine Gordon equations or more general nonlinear evolution systems. In Section 3 we show that (4) can be written as

$$
\left(\alpha^{*}-\alpha\right) a+\left(\beta^{*}-\beta\right) b+\left(\delta^{*}-\delta\right) c \geq 0, \quad q=(\alpha, \beta, \delta) \in P_{a d},
$$

where the constants $a, b, c$ are expressed through the solutions of the state and adjoint systems. This inequality is a necessary condition for the optimal set of parameters $q^{*}$. Furthermore, if

$$
P_{a d}=\left[\alpha_{1}, \alpha_{2}\right] \times\left[\beta_{1}, \beta_{2}\right] \times\left[\delta_{1}, \delta_{2}\right], \quad \beta_{1}>0
$$

and all of $a, b, c$ are not zeros, then the necessary condition (5) becomes

$$
\begin{aligned}
\alpha^{*} & =\frac{1}{2}\{\operatorname{sign}(a)+1\} \alpha_{2}-\frac{1}{2}\{\operatorname{sign}(a)-1\} \alpha_{1}, \\
\beta^{*} & =\frac{1}{2}\{\operatorname{sign}(b)+1\} \beta_{1}-\frac{1}{2}\{\operatorname{sign}(b)-1\} \beta_{1}, \\
\delta^{*} & =\frac{1}{2}\{\operatorname{sign}(c)+1\} \delta_{2}-\frac{1}{2}\{\operatorname{sign}(c)-1\} \delta_{1},
\end{aligned}
$$

which is a bang-bang control law.

The goals of this paper are to develop a spectral method for the solution of the state and the adjoint equations (1) and (29), apply the Powell's minimization method for the identification of the optimal parameters $q^{*}$ in $(3)$, and to examine the numerical applicability of the bang-bang control law (5).

The paper is organized as follows. In Section 2 we develop a spectral method for the solution of the state and adjoint equations (1) and (29). In Section 3 the adjoint system is introduced and the necessary condition (5) is established. In Section 4 we examine the parameter identification problem for (3) subject to (1) and introduce a modification of the Powell's minimization method. Finally, in Section 5 we discuss the numerical results. 


\section{Spectral method}

Let $I=(0, L)$ and $H_{0}^{r}(I)$ be the Sobolev space on $I$ with the norm $\|u\|_{r}$. Let the Hilbert space $H$ have the norm $|u|$ and the inner product $(u, v)$. When $r=1$, we denote the inner product in $H_{0}^{1}(I)$ by $((u, u))=(\nabla u, \nabla u)$, and its norm by $\|u\|$. Let $\langle u, v\rangle$ denote the duality pairing between $V=H_{0}^{1}(I)$ and $V^{\prime}=H^{-1}(I)$. Then we can define a self-adjoint operator $A$ with the domain $D(A)=H_{0}^{1}(I) \cap H^{2}(I)$ by the relation $\langle A u, v\rangle=((u, v))$, and $A u=-u_{x x}$ for $u \in D(A)$.

As in [2] the variational formulation for the weak solutions of (1) is given by

$$
\begin{aligned}
& \left\langle\frac{\partial^{2} y}{\partial t^{2}}, v\right\rangle+\alpha\left(\frac{\partial y}{\partial t}, v\right)+\beta((y, v))+\delta(\sin y, v)=(f(t), v), \quad v \in V, \\
& y(0)=y_{0} \text { and } \frac{\partial y}{\partial t}(0)=y_{1} .
\end{aligned}
$$

Problem (6) is an initial value problem for a formal abstract second-order differential equation in $H$ :

$$
\begin{aligned}
& y^{\prime \prime}+\alpha y^{\prime}+\beta A y+\delta \sin y=f(t), \quad t \in(0, T), \\
& y(0)=y_{0}, \quad y^{\prime}(0)=y_{1},
\end{aligned}
$$

where ${ }^{\prime}=d / d t$ and ${ }^{\prime \prime}=d^{2} / d t^{2}$. The weak solutions of (6) are the solutions of (7) sought in the Hilbert space

$$
W(0, T)=\left\{u \mid u \in L^{2}(0, T ; V), u^{\prime} \in L^{2}(0, T ; H), u^{\prime \prime} \in L^{2}\left(0, T ; V^{\prime}\right)\right\} .
$$

The existence and uniqueness results for the weak solutions of (7) are summarized in Theorem 2.1, see [4] for the proofs.

Theorem 2.1. Let $\alpha, \delta \in R, \beta>0$ and let us assume that

$$
y_{0} \in V, \quad y_{1} \in H, \quad \text { and } f \in L^{2}(0, T ; H) .
$$

Then there exists a unique weak solution $y \in L^{2}(0, T ; V)$ of $(7)$. This solution satisfies $y \in C([0, T] ; V) \cap W(0, T), y^{\prime} \in C([0, T] ; H)$, and

$$
\|y(t)\|^{2}+\left|y^{\prime}(t)\right|^{2} \leq C_{1}\left[\left\|y_{0}\right\|^{2}+\left|y_{1}\right|^{2}+\|g\|_{L^{2}(0, T ; H)}^{2}\right], \quad t \in[0, T]
$$

where $C_{1}$ is a constant.

Let us introduce the spectral method in order to solve the state and adjoint state equations. To accommodate the zero boundary conditions in (1) and (29) functions $e_{n}(x)=\sin (\pi n x / L), n=1,2, \ldots$ are chosen as a (non-normalized) basis in $H=L_{2}(I)$. Let $S_{N}$ be the subspace of $H$ spanned by the sine functions $\left\{e_{n}(x)\right\}_{n=1}^{N}$. Let $P_{N}$ be the projection operator onto $S_{N}$ in $H$. Let $y_{N}(t)=$ $y_{N}(\cdot, t) \in S_{N}$ be the solution of

$$
\begin{gathered}
\left(\frac{\partial^{2} y_{N}}{\partial t^{2}}, v\right)+\alpha\left(\frac{\partial y_{N}}{\partial t}, v\right)+\beta\left(\left(y_{N}, v\right)\right)+\delta\left(\sin y_{N}, v\right)=(f(t), v), \\
v \in S_{N}, \quad t \in(0, \\
\left(\left(y_{N}(0)-y(0), v\right)\right)=0, \quad\left(\frac{\partial y_{N}}{\partial t}(0)-y_{1}, v\right)=0, \quad v \in S_{N} .
\end{gathered}
$$


We need the following well-known error estimate [9]: for any $s, r \in(-\infty, \infty)$ with $0 \leq s \leq r$,

$$
\left\|P_{N} u-u\right\|_{s} \leq C_{2}\left(1+N^{2}\right)^{(s-r) / 2}\|u\|_{r} \text { for } u \in H_{0}^{r}(I),
$$

where $P_{N}: H \rightarrow S_{N}$ is the projection operator, and $C_{2}$ is a constant dependent on $L$. Using $P_{N}$ the initial value problem (11) can be written in an equivalent form

$$
\begin{aligned}
& y_{N}^{\prime \prime}+\alpha y_{N}^{\prime}+\beta A y_{N}+\delta P_{N} \sin y_{N}=P_{N} f(t), \quad t \in(0, T), \\
& y_{N}(0)=P_{N} y_{0}, \quad y_{N}^{\prime}(0)=P_{N} y_{1} .
\end{aligned}
$$

Now we establish error bounds for finite spectral approximations $y_{N}(t)$.

Theorem 2.2. Let $r>0$. If the solution $y$ of (7) satisfy $y \in H_{0}^{r}(I)$, then there is a $C_{3}$ such that

$$
\left|y(t)-y_{N}(t)\right| \leq C_{3}\left(1+N^{2}\right)^{-r / 2}, \quad t \in[0, T] .
$$

Proof. Setting $Y_{N}=P_{N} y$ and applying the projection operator $P_{N}$ to $(7)$ we get

$$
\begin{aligned}
& Y_{N}^{\prime \prime}+\alpha Y_{N}^{\prime}+\beta A Y_{N}+\delta P_{N} \sin y=P_{N} f(t), \quad t \in(0, T), \\
& Y_{N}(0)=P_{N} y_{0}, \quad Y_{N}^{\prime}(0)=P_{N} y_{1} .
\end{aligned}
$$

Indeed, the first equation of (7) is changed into

$$
\frac{d}{d t}\left[y^{\prime}(t)+\beta A \int_{0}^{t} y(s) d s\right]=f(t)-\delta \sin y(t)-\alpha y^{\prime}(t) .
$$

Since the function in $[\cdots]$ and the right side term of (15) belong to $H$, it follows that $\frac{d}{d t}[\cdots] \in H$. Since $\left(\frac{\partial u}{\partial t}, e_{n}\right)=\frac{d}{d t}\left(u, e_{n}\right)$ for $u \in H$, we have

$$
\frac{d}{d t} P_{N}\left[y^{\prime}(t)+\beta A \int_{0}^{t} y(s) d s\right]=P_{N}\left[f(t)-\delta \sin y(t)-\alpha y^{\prime}(t)\right] .
$$

Furthermore by the linearity of $P_{N}$ we have

(17) $\frac{d}{d t}\left[\left(P_{N} y\right)^{\prime}(t)+\beta A P_{N} \int_{0}^{t} y(s) d s\right]=P_{N} f(t)-\delta P_{N} \sin y(t)-\alpha\left(P_{N} y\right)^{\prime}(t)$.

Here we used the fact that $A$ and $P_{N}$ are commutable on $H$. Differentiating the left side of (17) we get

$$
\left(P_{N} y\right)^{\prime \prime}(t)+\beta A\left(P_{N} y\right)(t)=P_{N} f(t)-\delta P_{N} \sin y(t)-\alpha\left(P_{N} y\right)^{\prime}(t) .
$$

Subtracting (14) from (13) and setting $U_{N}=y_{N}-Y_{N}$ we obtain

$$
\begin{aligned}
& U_{N}^{\prime \prime}+\alpha U_{N}^{\prime}+\beta A U_{N}=\delta P_{N}\left(\sin y-\sin y_{N}\right), \quad t \in(0, T), \\
& U_{N}(0)=0, \quad U_{N}^{\prime}(0)=0 .
\end{aligned}
$$


Multiplying (19) by $2 U_{N}^{\prime}$ and using differential properties of the inner product, we get

(20)

$\frac{d}{d t}\left|U_{N}^{\prime}(t)\right|^{2}+2 \alpha\left|U_{N}^{\prime}(t)\right|^{2}+\beta \frac{d}{d t}\left\|U_{N}(t)\right\|^{2}=2 \delta\left(P_{N}\left(\sin y(t)-\sin y_{N}(t)\right), U_{N}^{\prime}(t)\right)$.

Integrating (19) over $[0, t]$ by parts, we have

$$
\begin{aligned}
& \left|U_{N}^{\prime}(t)\right|^{2}+2 \alpha \int_{0}^{t}\left|U_{N}^{\prime}(s)\right|^{2} d s+\beta\left\|U_{N}(t)\right\|^{2} \\
= & 2 \delta \int_{0}^{t}\left(P_{N}\left(\sin y(s)-\sin y_{N}(s)\right), U_{N}^{\prime}(s)\right) d s .
\end{aligned}
$$

Now let us estimate the right side term of (21).

$$
\begin{aligned}
& 2 \int_{0}^{t}\left|\left(P_{N}\left(\sin y(s)-\sin y_{N}(s)\right), U_{N}^{\prime}(s)\right)\right| d s \\
\leq & 2 \int_{0}^{t}\left\|P_{N}\right\|\left|\sin y(s)-\sin y_{N}(s)\right|\left|U_{N}^{\prime}(s)\right| d s \\
\leq & 2 \int_{0}^{t}\left(|y(s)-Y(s)|+\left|U_{N}(s)\right|\right)\left|U_{N}^{\prime}(s)\right| \\
\leq & \int_{0}^{t}|y(s)-Y(s)|^{2} d s+\int_{0}^{t}\left[k^{2}\left\|U_{N}(s)\right\|^{2} d s+\left|U_{N}^{\prime}(s)\right|^{2}\right] d s \\
\leq & C_{2}\left(1+N^{2}\right)^{-r}\|y\|_{L^{2}(0, T ; H)}^{2}+\int_{0}^{t}\left[k^{2}\left\|U_{N}(s)\right\|^{2}+\left|U_{N}^{\prime}(s)\right|^{2}\right] d s .
\end{aligned}
$$

Letting $\tilde{\beta}=\min \{1, \beta\}, \tilde{k}=\max \left\{1, k^{2}\right\}, k_{\beta}=\tilde{k} / \tilde{\beta}$ and $C_{\beta}=C_{2} / \tilde{\beta}$ and substituting the above result into (21), we obtain

$$
\begin{aligned}
& \left|U_{N}^{\prime}(t)\right|^{2}+\left\|U_{N}(t)\right\|^{2} \\
\leq & C_{\beta}\left(1+N^{2}\right)^{-r}\|y\|_{L^{2}(0, T ; H)}^{2}+k_{\beta} \int_{0}^{t}\left[\left\|U_{N}(s)\right\|^{2}+\left|U_{N}^{\prime}(s)\right|^{2}\right] d s .
\end{aligned}
$$

Applying Bellman-Gronwall Lemma to (22) we get

$$
\left|U_{N}^{\prime}(t)\right|^{2}+\left\|U_{N}(t)\right\|^{2} \leq C_{\beta}\left(1+N^{2}\right)^{-r}\|y\|_{L^{2}(0, T ; H)}^{2} e^{k_{\beta} T} .
$$

Now it follows from (12) and (23) that

$$
\begin{aligned}
\left|y(t)-y_{N}(t)\right| & \leq\left|y(t)-Y_{N}(t)\right|+\left|U_{N}(t)\right| \\
& \leq\left(1+N^{2}\right)^{-r / 2}\left[C_{2}\|y(t)\|_{r}+C_{\beta} k^{-1}\|y\|_{L^{2}(0, T ; H)} e^{k_{\beta} T / 2}\right] .
\end{aligned}
$$

Finally the conclusion is deduced from Theorem 2.1.

Expanding the functions in (11) into the Fourier sine series, and using $v=$ $\mathrm{u}_{k}, k=1,2, \ldots, N$ there we get

$$
\begin{aligned}
& Y_{k}^{\prime \prime}+\alpha Y_{k}^{\prime}+\beta_{k} Y_{k}+\delta S_{k}(t)=F_{k}(t), t \in(0, T), \\
& Y_{k}(0)=Y_{k_{0}}, \quad Y_{k}^{\prime}(0)=Y_{k_{1}},
\end{aligned}
$$


where $\beta_{k}=\beta k^{2} \pi^{2} / L^{2}, S_{k}(t)$ is the $k$-th Fourier sine coefficient of $P_{N} \sin y_{N}(t)$, and $Y_{k}(t), F_{k}(t), Y_{k_{0}}$, and $Y_{k_{1}}$ are the Fourier coefficients of the solution $y_{N}(t)$ and the corresponding functions in (11). Thus the approximate solutions of (1) are given by

$$
y_{N}(t, x)=\sum_{n=1}^{N} Y_{n}(t) e_{n}(x) .
$$

Similarly one can define the approximate solutions $w_{N}(t) \in S_{N}$ of the adjoint system (29) by

$$
w_{N}(t, x)=\sum_{n=1}^{N} W_{n}(t) e_{n}(x),
$$

where

$$
\begin{aligned}
& W_{k}^{\prime \prime}-\alpha W_{k}^{\prime}+\beta_{k} W_{k}+\delta C_{k}(t) W_{k}=Y_{k}(t)-Z_{k}(t), t \in(0, T), \\
& W_{k}(T)=0, \quad W_{k}^{\prime}(T)=0
\end{aligned}
$$

and $C_{k}(t)$ is the $k$-th Fourier sine coefficient of $P_{N} \cos y_{N}(t)$ and $Z_{k}(t)$ is the $k$-th Fourier sine coefficient of $P_{N} z_{d}(t)$.

\section{Optimal parameters}

In this section we show the continuity and the Gâteaux differentiability of the objective function $J(q)$, as well as establish the necessary condition (5) for an optimal set of coefficients $q^{*}$.

According to Theorem 2.1 given $q \in P_{a d}$ the weak solution $y(q)$ of $(7)$ is an element of the space $W(0, T)$ introduced in (8). By Theorem 3.1 in [5] the solution map $q \rightarrow y(q): P_{a d} \rightarrow W(0, T)$ is weakly continuous. In particular, if $q_{n} \rightarrow q$ as $n \rightarrow \infty$ in $P_{a d} \subset \mathbb{R}^{3}$, then $y\left(q_{n}\right) \rightarrow y(q)$ weakly in $L^{2}(0, T ; V)$. Since $V=H_{0}^{1}(I)$ is compactly imbedded in $H=L^{2}(I)$ it follows that $y\left(q_{n}\right) \rightarrow y(q)$ strongly in $L^{2}(0, T ; H)$. Thus the objective function $J(q)=\left\|y(q)-z_{d}\right\|_{L^{2}(0, T ; H)}^{2}$ is continuous on $P_{a d}$.

In fact, we can show that $J(q)$ is Gâteaux differentiable. By Theorem 3.3 in [5] the map $q \rightarrow y(q)$ is weakly Gâteaux differentiable at $q^{*}$ in the direction of $q-q^{*}$. The proof is based on the transposition method of J. Lions, see [5] for details. According to this theorem the weak Gâteaux derivative $D y\left(q^{*}\right)\left(q-q^{*}\right)$ of $y(q)$ at $q^{*}$ in the direction of $q-q^{*}$ can be described as follows.

Let $q=(\alpha, \beta, \delta)$ and $q^{*}=\left(\alpha^{*}, \beta^{*}, \delta^{*}\right)$. For $u \in W(0, T)$ with $u(T, x)=$ $0, u_{t}(T, x)=0, x \in(0, L)$ and $u(t, 0)=u(t, L)=0, t \in(0, T)$ define the linear operator $\mathcal{L}$ by

$$
\mathcal{L}(u)=u^{\prime \prime}-\alpha^{*} u^{\prime}-\beta^{*} u_{x x}+\delta^{*} \cos y\left(q^{*}\right) u
$$

Let

$$
X_{0}=\left\{u \in W(0, T): \mathcal{L}(u)=g, u(T)=u_{t}(T)=0, g \in L^{2}(0, T ; H)\right\} .
$$


That is $X_{0}$ is the collection of the weak solutions $u$ of

$$
\begin{aligned}
& u_{t t}-\alpha^{*} u_{t}-\beta^{*} u_{x x}+\delta^{*} \cos y\left(q^{*}\right) u=g(t, x), \quad(t, x) \in Q, \\
& u(t, 0)=u(t, L)=0, \quad t \in(0, T), \\
& u(T, x)=0, \quad u_{t}(T, x)=0, \quad x \in(0, L) .
\end{aligned}
$$

Then the weak Gâteaux derivative $D y\left(q^{*}\right)\left(q-q^{*}\right)$ is the function $z=z\left(q, q^{*}\right) \in$ $L^{2}(0, T ; H)$ that is the unique solution of the integral equation

$$
\begin{aligned}
\int_{Q} z(t, x) \mathcal{L}(v)(t, x) d x d t= & \left(\alpha^{*}-\alpha\right) \int_{Q} y_{t}\left(q^{*} ; t, x\right) v(t, x) d x d t \\
& +\left(\beta^{*}-\beta\right) \int_{Q} y_{x}\left(q^{*} ; t, x\right) v_{x}(t, x) d x d t \\
& +\left(\delta^{*}-\delta\right) \int_{Q} \sin y\left(q^{*} ; t, x\right) v(t, x) d x d t
\end{aligned}
$$

for any $v \in X_{0}$.

From the definition of the functional $J(q)=\left\|y(q)-z_{d}\right\|_{L^{2}(0, T ; H)}^{2}$

$$
\begin{aligned}
\operatorname{DJ}\left(q^{*}\right)\left(q-q^{*}\right) & =2\left(y\left(q^{*}\right)-z_{d}, D y\left(q^{*}\right)\left(q-q^{*}\right)\right) \\
& =2 \int_{Q}\left[y\left(q^{*} ; t, x\right)-z_{d}(t, x)\right] z(t, x) d x d t .
\end{aligned}
$$

Thus the necessary condition (4) is

$$
\int_{Q}\left[y\left(q^{*} ; t, x\right)-z_{d}(t, x)\right] z(t, x) d x d t \geq 0, \quad q \in P_{a d} .
$$

Now let $w \in X_{0}$ be the adjoint state. That is $\mathcal{L}(w)=y\left(q^{*}\right)-z_{d}$ or

$$
\begin{aligned}
& w_{t t}-\alpha^{*} w_{t}-\beta^{*} w_{x x}+\delta^{*} \cos y\left(q^{*}\right) w=y\left(q^{*}\right)-z_{d} \text { in } Q, \\
& w(t, 0)=w(t, L)=0, \quad t \in(0, T) \\
& w(T, x)=0, \quad w_{t}(T, x)=0, \quad x \in(0, L) .
\end{aligned}
$$

Using $w$ in place of $v$ in (27) allows us to rewrite condition (28) as (30) $\quad\left(\alpha^{*}-\alpha\right) a+\left(\beta^{*}-\beta\right) b+\left(\delta^{*}-\delta\right) c \geq 0, \quad q=(\alpha, \beta, \delta) \in P_{a d}$, where

$$
\begin{aligned}
a & =\int_{Q} y_{t}(t, x) w(t, x) d x d t, \\
b & =\int_{Q} y_{x}(x, t) w_{x}(t, x) d x d t, \\
c & =\int_{Q} \sin y(t, x) w(t, x) d x d t .
\end{aligned}
$$

Thus the inequality (30) is a necessary condition for the optimal set $q^{*}$. In case the admissible set $P_{a d}$ is a box in $\mathbb{R}^{3}$ one uses the fact that (30) must 
be satisfied for any $q=(\alpha, \beta, \delta) \in P_{a d}$ to derive the bang-bang control law mentioned in the Introduction.

\section{Identification problem}

Since our goal is to give a numerical solution to the minimization problem (3) the objective function $J(q)$ introduced in (2) is replaced with its time-discrete approximation (still named $J$ ):

$$
J(q)=\sum_{j=1}^{M}\left|y\left(q ; t_{j}\right)-z_{d}\left(t_{j}\right)\right|^{2}, \quad z_{d}\left(t_{j}\right) \in H, q \in P .
$$

The approximate cost functional corresponding to (31) will be given by

$$
J_{N}(q)=\sum_{j=1}^{M}\left|y_{N}\left(q ; t_{j}\right)-z_{d}\left(t_{j}\right)\right|^{2}, \quad q \in P,
$$

where $y_{N}(q)$ is the weak solution of (11). The parameter identification problem for (32) is to find $q_{N}^{*} \in P_{a d}$ such that

$$
J_{N}\left(q_{N}^{*}\right)=\min _{q \in P_{a d}} J_{N}(q) .
$$

As in [5], one can easily prove that the cost functional (33) is continuous on $P_{a d}$. Therefore the minimization problem admits a minimum in $P_{a d}$.

Lemma 4.1. There exists $C_{4}>0$ independent on $N$ such that

$$
\left|J_{N}(q)-J(q)\right| \leq C_{4}\left(1+N^{2}\right)^{-r}
$$

Proof. Using the orthogonality between $y(q)-y_{N}(q)$ and $S_{N}$, one has at $t=t_{j}$ :

$$
\begin{aligned}
\left|y_{N}(q)-z_{d}\right|^{2}-\left|y(q)-z_{d}\right|^{2} & =\left(y_{N}(q)-y, y_{N}(q)+y(q)-2 z_{d}\right) \\
& =\left(y_{N}(q)-y, y(q)-y_{N}(q)-2 z_{d}\right) .
\end{aligned}
$$

Hence by Theorem 2.2 we have

$$
\begin{aligned}
\left|J_{N}(q)-J(q)\right| & \leq \sum_{j=1}^{M}\left|y_{N}\left(q ; t_{j}\right)-y\left(q ; t_{j}\right)\right|^{2}+2 \sum_{j=1}^{M}\left|y_{N}\left(q ; t_{j}\right)-y\left(q ; t_{j}\right)\right|\left|z_{d}\left(t_{j}\right)\right| \\
& \leq\left[C_{3}^{2} M+2 C_{3} \sum_{j=1}^{M}\left|z_{d}\left(t_{j}\right)\right|\right]\left(1+N^{2}\right)^{-r / 2},
\end{aligned}
$$

where $C_{4}=C_{3}^{2} M+2 C_{3} \sum_{j=1}^{M}\left|z_{d}\left(t_{j}\right)\right|$.

Theorem 4.2. Let $\left\{q_{N}^{*}\right\}$ be a sequence satisfying (33) and $q^{*}$ be its limit point. Then $J\left(q^{*}\right)=\min _{q \in P_{a d}} J(q)$. 
Proof. Let $\left\{q_{N_{k}}^{*}\right\}$ be a subsequence of $\left\{q_{N}^{*}\right\}$ such that $q_{N_{k}}^{*} \rightarrow q^{*}$. Since

$$
\left|J_{N_{k}}\left(q_{N_{k}}^{*}\right)-J\left(q^{*}\right)\right| \leq\left|J_{N_{k}}\left(q_{N_{k}}^{*}\right)-J\left(q_{N_{k}}^{*}\right)\right|+\left|J\left(q_{N_{k}}^{*}\right)-J\left(q^{*}\right)\right|,
$$

it follows from Lemma 4.1 and the continuity of $y(q)$ that $\lim _{k \rightarrow \infty} J_{N_{k}}\left(q_{N_{k}}^{*}\right)=$ $J\left(q^{*}\right)$. Let $\bar{q}$ be such that $J(\bar{q})=\min _{q \in P_{a d}} J(q)$. Since $J(\bar{q})=\lim _{N \rightarrow \infty} J_{N}(\bar{q})$ and $J_{N}\left(q_{N}^{*}\right) \leq J_{N}(\bar{q})$, we have $J\left(q^{*}\right) \leq J(\bar{q})$. Therefore we conclude that $J(\bar{q})=J\left(q^{*}\right)$.

Let $z_{d}(t)=P_{N} z_{d}(t)=\sum_{n=1}^{N} Z_{n}(t) e_{n}$. Then the cost functional $J_{N}(q)$ is

$$
J_{N}(q)=\frac{L}{2} \sum_{n=1}^{N} \sum_{j=1}^{M}\left[Y_{n}\left(q ; t_{j}\right)-Z_{n}\left(t_{j}\right)\right]^{2}, \quad q \in P_{a d},
$$

where $Y_{k}(q ; t)$ is the solution $Y_{k}(t)$ of $(24)$ for the given values of the parameters $q=(\alpha, \beta, \delta) \in P_{a d}$. Lemma 4.1 and Theorem 4.2 hold for the cost functional (34).

The minimization problem for $J_{N}(q)$ is solved using a modification of Powell's minimization method, see [3]. the advantage of the Powell's method is that it does not require the knowledge of the derivatives (gradients) to minimize functions of several variables, but it still achieves a quadratic convergence near the points of minima.

The method iteratively minimizes a function of $K$ variables (here $K=3$ ). Steps 1-7 describe one iteration of the method.

(1) Initialize the set of directions $u_{i} \in \mathbb{R}^{3}$ to the standard basis vectors $\mathrm{e}_{i}$ in $\mathbb{R}^{3}$

$$
u_{i}=\mathrm{e}_{i}, \quad i=1,2,3 .
$$

(2) Save your starting position as $q_{0} \in \mathbb{R}^{3}$.

(3) For $i=1,2,3$ move from $q_{0}$ along the direction $u_{i}$ and find the point of minimum $p_{i}$.

(4) Re-index the directions $u_{i}$, so that (for the new indices) $J_{N}\left(p_{1}\right) \leq$ $J_{N}\left(p_{2}\right) \leq J_{N}\left(p_{3}\right) \leq J_{N}\left(q_{0}\right)$.

(5) Move from $q_{0}$ along the new direction $u_{1}$ and find the point of minimum $r_{1}$. Move from $r_{1}$ along the direction $u_{2}$ and find the point of minimum $r_{2}$. Move from $r_{2}$ along the direction $u_{3}$ and find the point of minimum $r_{3}$.

(6) Set $v=r_{3}-q_{0}$.

(7) Move from $q_{0}$ along the direction $v$ and find the minimum. Call it $q_{0}$. It replaces $q_{0}$ from step 2.

(8) Repeat the above steps until a stopping criterion is satisfied.

If the minimization is to be restricted to a subset $P_{a d} \subset \mathbb{R}^{K}$, then the moves in the one-dimensional minimization steps above are restricted so that the trial points would not leave $P_{a d}$. 


\section{Numerical results}

In all the numerical experiments we used the values specified in Table 1, and observation times $t_{j}=(T / M) j, j=1,2, \ldots, M$.

TABLE 1. Parameter values for numerical simulations

\begin{tabular}{ll}
\hline Time and spatial intervals & {$[0, T] \times[0, L]=[0,4] \times[0, \pi]$} \\
Admissible set & $P_{a d}=[0.001,1] \times[0.1,1] \times[0.1,1]$ \\
Initial conditions & $y_{0}(x)=0$ \\
& $y_{1}(x)=\exp \left[-100(x-\pi / 2)^{2}\right]$ \\
Forcing function & $f(t, x)=0.01$ \\
$N$ & 16 \\
\hline
\end{tabular}

To simulate the data $z_{d}(t, x)$ let $\hat{q} \in P_{a d}$ be the test set of parameters. The initial value problems (24) and (25) were solved using a Leap-Frog Method with the time step $h=0.01$ as follows. Define $Y_{k}^{j}, k=1,2, \ldots, N$ by

$$
\begin{aligned}
& Y_{k}^{-1}=Y_{k_{0}}-h Y_{k_{1}}, \\
& Y_{k}^{j+1}=\frac{2 Y_{k}^{j}-\left[\beta_{k} Y_{k}^{j}-F_{k}\left(t_{j}\right)+\delta S_{k}\left(t_{j}\right) h^{2}\right]+(1-\alpha h / 2) Y_{k}^{j-1}}{1+\alpha h / 2}
\end{aligned}
$$

for $j=0,1,2, \ldots$. Then $Y_{k}^{j}$ is an approximation of $Y_{k}(t)$ at $t=t_{j}=h j$.

Since real data always contain some noise, we set

$$
z_{d}(t, x)=y(\hat{q} ; t, x)+\epsilon \eta(x),
$$

where $\eta(x)$ is a random variable uniformly distributed on interval $[-1,1]$, and $\epsilon$ is a small constant.

Let $q_{0} \in P_{a d}$ be an arbitrarily chosen set of parameters. The Powell's minimization method iteratively generates the sequence $q_{1}, q_{2}, \ldots$ as described in Section 3. The stopping criterion for this iterative process was chosen to be

$$
\frac{\left|J_{N}\left(q_{m}\right)-J_{N}\left(q_{m-1}\right)\right|}{\left|J_{N}\left(q_{0}\right)\right|}<10^{-6} \text {. }
$$

Let us consider $\hat{q}=(0.02,1,0.5)$ which is a typical point in $P_{a d}$. Let $q_{N}^{*}=$ $q_{m}$ be the set of parameters attained when the Powell's minimization method was terminated according to the stopping criterion (36). The minimizers $q_{N}^{*}$ together with the number of iterations $m$ are shown in Tables 2, 3 and 4 for various noise levels $\epsilon$, and the number of observations $M$.

Table 2 shows the identification algorithm is successful without noise. It can be seen that the number of iterations is quite small and the precision of the identification is high even for a few time observation data. Tables 3, 4 show that significant time observation data is required when noise is present. 
TABLE 2. Identification results for $\epsilon=0$

\begin{tabular}{cccc}
\hline$M$ & Iterations $(m)$ & $q_{N}^{*}$ & $J_{N}\left(q_{N}^{*}\right)$ \\
\hline 1 & 4 & $(0.01983,1.00000,0.49997)$ & 0.000000 \\
4 & 4 & $(0.01943,0.99999,0.50004)$ & 0.000000 \\
10 & 4 & $(0.01959,0.99999,0.50005)$ & 0.000000 \\
400 & 4 & $(0.02000,1.00000,0.50000)$ & 0.000000 \\
\hline
\end{tabular}

TABLE 3. Identification results for $\epsilon=0.01$

\begin{tabular}{cccc}
\hline$M$ & Iterations & $q_{N}^{*}$ & $J_{N}\left(q_{N}^{*}\right)$ \\
\hline 1 & 20 & $(1.00000,0.60011,0.10000)$ & 0.000012 \\
4 & 3 & $(0.00100,1.00000,0.10000)$ & 0.000064 \\
10 & 6 & $(0.10379,1.00000,0.81481)$ & 0.000161 \\
400 & 4 & $(0.01981,0.99998,0.50071)$ & 0.006171 \\
\hline
\end{tabular}

TABLE 4. Identification results for $\epsilon=0.1$

\begin{tabular}{cccc}
\hline$M$ & Iterations & $q_{N}^{*}$ & $J_{N}\left(q_{N}^{*}\right)$ \\
\hline 400 & 4 & $(0.01810,0.99981,0.50712)$ & 0.617093 \\
\hline
\end{tabular}

Now let us evaluate the values $a, b, c$. For $t_{j}=j h, h>0$ put

$$
\begin{aligned}
a_{N} & =\int_{0}^{T} \int_{0}^{L} \frac{\partial y_{N}}{\partial t}(t, x) w_{N}(t, x) d x d t \\
& =\frac{L}{2} \sum_{n=1}^{N} \int_{0}^{T} Y_{n}^{\prime}(t) W_{n}(t) d t \\
& \approx \frac{L h}{2} \sum_{n=1}^{N} \sum_{j=1}^{M} Y_{n}^{\prime}\left(t_{j}\right) W_{n}\left(t_{j}\right)=a_{N M}, \\
& =\int_{0}^{T} \int_{0}^{L} \frac{\partial y_{N}}{\partial x}(t, x) \frac{\partial w_{N}}{\partial x}(t, x) d x d t \\
& =\frac{\pi^{2}}{2 L} \sum_{n=1}^{N} n^{2} \int_{0}^{T} Y_{n}(t) W_{n}(t) d t \\
& \frac{\pi^{2} h}{2 L} \sum_{n=1}^{N} n^{2} \sum_{j=1}^{M} Y_{n}\left(t_{j}\right) W_{n}\left(t_{j}\right)=b_{N M},
\end{aligned}
$$


and

$$
\begin{aligned}
c_{N} & =\int_{0}^{T} \int_{0}^{L} P_{N}\left[\sin y_{N}(t, x)\right] w_{N}(t, x) d x d t \\
& =\frac{L}{2} \sum_{n=1}^{N} \int_{0}^{T} S_{n}(t) W_{n}(t) d t \\
& \approx \frac{L h}{2} \sum_{n=1}^{N} \sum_{j=1}^{M} S_{n}\left(t_{j}\right) W_{n}\left(t_{j}\right)=c_{N M} .
\end{aligned}
$$

It is clear that

$$
\lim _{N, M \rightarrow \infty} a_{N, M}=a, \lim _{N, M \rightarrow \infty} b_{N, M}=b, \lim _{N, M \rightarrow \infty} c_{N, M}=c .
$$

For the interior point $q^{*}=(0.02,0.7,0.5)$ of $P_{a d}$, the estimated values are

$a_{16,400}=0.360151 \times 10^{-8}, b_{16,400}=-0.103648 \times 10^{-5}, c_{16,400}=0.473936 \times 10^{-13}$.

For the boundary point $q^{*}=(0.001,1,0.1)$ of $P_{a d}$, the estimated values are

$a_{16,400}=-0.04369 \times 10^{-8}, b_{16,400}=0.188047 \times 10^{-6}, c_{16,400}=0.208795 \times 10^{-8}$.

All estimated values can be regarded as zero. It they are not, i.e., $b_{16,400}=$ $-0.103648 \times 10^{-5} \not \approx 0$, then $\beta^{*}$ must be 1 by the bang-bang control law. So, we can conclude that the bang-bang control law was not useful in our numerical experiments.

\section{References}

[1] A. R. Bishop, K. Fesser, and P. S. Lomdahl, Influence of solitons in the initial state on chaos in the driven damped sine-Gordon system, Phys. D 7 (1983), no. 1-3, 259-279.

[2] R. Dautary and J. L. Lions, Mathematical Analysis and Numerical Methods for Science and Technology, Vol. 5, Evolution Problems I, Springer-Verlag, 1992.

[3] S. Gutman, Identification of piecewise-constant potentials by fixed-energy phase shifts, Appl. Math. Optim. 44 (2001), no. 1, 49-65.

[4] J. Ha and S. Nakagiri, Existence and regularity of weak solutions for semilinear second order evolution equations, Funkcial. Ekvac. 41 (1998), no. 1, 1-24.

[5] _ Identification problems of damped sine-Gordon equations with constant parameters, J. Korean Math. Soc. 39 (2002), no. 4, 509-524.

[6] _ Identification problems for the system governed by abstract nonlinear damped second order evoution equations, J. Korean Math. Soc. 41 (2004), no. 3, 435-459.

[7] _ Identification of constant parameters in perturbed sin-Gordon equations, J. Korean Math. Soc. 43 (2006), no. 5, 931-950.

[8] M. Levi, Beating modes in the Josephson junction, Chaos in nonlinear dynamical systems (Research Triangle Park, N.C., 1984), 56-73, SIAM, Philadelphia, PA, 1984.

[9] B. Mercier, An Introdution to the Numerical Analysis of Spectral Methods, Lecture Notes in Physis 318, Springer-Verlag 1989.

[10] W. H. Press, S. A. Teukolsky, W. T. Vetterling, and B. P. Flannery, Numerical Recepies in FORTRAN (2nd Ed.), Cambridge University Press, Cambridge.

[11] R. Temam, Infinite-Dimensional Dynamical Systems in Mechanics and Physics, 2nd ed., Applied Mathematical Sciences, Vol. 68, Springer-Verlag, 1997. 
JUNHONG HA

School of Liberal Arts

Korea University of TeChnology and Education

Cheonan 330-708, Korea

E-mail address: hjh@kut.ac.kr

Semion Gutman

DePARTMENT OF MATHEMATics

UNIVERSITY OF OKLAHOMA

NORMAN, OK 73019, USA

E-mail address: sgutman@ou.edu 